\title{
The applicability of Napping in the analysis of fermented foods and beverages - A review
}

\author{
MÁRTON ISTVÁN and ZOLTÁN KÓKAI*
}

Department of Postharvest, Supply Chain, Commerce and Sensory Science, Institute of Food Technology and Food Science, Hungarian University of Agriculture and Life Sciences, Budapest, Hungary

\section{CONFERENCE FULL PAPER}

Received: May 5, 2021 • Accepted: August 11, 2021

Published online: September 1, 2021

(C) 2021 The Author(s)

\begin{abstract}
Napping is one of the rapid sensory profiling methods, which was established recently to meet the needs of sensory and consumer researchers. This approach provides a holistic evaluation of the tested sample through their positioning in a 2-dimensional space. The protocol of the analysis is somewhat different from the traditionally applied descriptive methods, like Quantitative Descriptive Analysis. In our review, we focus on the applicability of Napping in the field of fermented goods. The accompanying procedures are also investigated (typically Flash Profiling, CATA, and further methods), in order to understand how the combined datasets facilitate the understanding of the sensory characteristics of the products.
\end{abstract}

\section{KEYWORDS}

Napping, sensory analysis, fermented food, wine, beer

\section{INTRODUCTION}

Sensory analysis methods of food and beverage products might be classified many ways. A possible methodological distinction is related to the outcome of the tests: discrimination or characterization of the samples. During the evolvement of the methods there were several key issues related to the feasibility of the protocols. These are mostly the size and the skills of the panel, the time

*Corresponding author. E-mail: kokai.zoltan@uni-mate.hu 
requirement and the interpretation of the results. In the food industry, there is a growing demand for such methods that are simpler to implement, rapid and might be performed by untrained panelists (consumers) also. These rapid methods appeared from the late 1990's, including free choice profiling or flash profiling. The basis of these procedures rely on standard approaches (e.g. profile analysis or Quantitative Descriptive Analysis), but the necessary steps were simplified or reduced in order to achieve a faster method. Altogether, with the development of statistical procedures the number of available product characterization methods are increasing. In our present review, we analyze the applicability of one of these rapid methods: Napping. Jerome Pagés created napping in 2003. The name of the method originates from the French word 'nappe', which means tablecloth. The original idea of the method is to position the samples on a large piece of paper. The vicinity or the distance among the samples represent the similarity or the difference between their natures. This type of evaluation is often referred to as being holistic, analyzing the sensory stimuli of the samples as a whole, not focusing on the details.

The method's predecessor is projective mapping, used in psychology to examine the personality of individuals. Based on a psychological study, in 1994 Risvik asked sensory assessment participants to rank the chocolate samples they were testing on a scale according to their similarity and dissimilarity (separately). Risvik assessed the results primarily on the basis of the objective similarities and differences between the samples, without taking into account the subjective judgements of the panel members (Risvik et al., 1994).

However, the method developed by Pagés leaves it entirely up to the assessor to determine the similarities and differences. Another significant difference from Risvik's method is that it requires the assessors to place the samples in a 2-dimensional space rather than on a 1dimensional scale, thus providing a more detailed picture of the assessors' perception of the products. According to Pagés, traditional sensory methods do not necessarily look at the qualities that consumers consider important. In other words, the aim of Napping is to identify the most important similarities and differences between the samples tested. Based on the dimensions of the "nappe" used (typical $40^{\star} 60 \mathrm{~cm}$ ), the participants in the study place the samples along the longer side according to the aspect they consider more important and along the shorter side according to the less important aspect (Pagés, 2005).

In previous studies, the evaluation of results was distorted and complicated by the need to sort the results of one-dimensional scales into two-dimensional matrices, and further difficulties were caused when assessors had to judge similarities and differences between a larger number of samples $(>10)$, as they often tested those in groups or pairs. The method developed by Pagés, however, eliminated these errors, as assessors sort the entire sample set into a two-dimensional matrix and rate the samples simultaneously (Pagés, 2005).

Furthermore, the evaluation of the resulting dataset also plays a significant role. The typical statistical method used to evaluate the results of the study is the Multiple Factor Analysis (MFA), which was also used by Pagés in his paper. MFA is a method developed by Escofier and Pagés in which variables are grouped into different blocks during the analysis (Escofier and Pages, 1994). MFA is performed in two consecutive steps. First, a PCA (Principal Component Analysis) is performed on the members of each block, and then the members of the blocks are "normalized" by dividing the member by the square root of the value of the first principal component. The resulting "normalised" values are then placed in a matrix and another PCA is performed on the entire matrix. The original data set is then applied to the analyzed data and similarities and differences are compared (Abdi and Valentin, 2007). 
In our present review, we analyze the applicability of Napping on fermented food and beverage products based on previous research articles in this field.

\section{MATERIALS AND METHODS}

We performed a detailed literature search using the keywords 'Napping', 'sensory' and some further expressions, which were related to the samples, originated from fermentation technologies. These were typically: wine, beer, cheese and yogurt. Our goal was to provide a detailed and analytical overview of the method for researchers and sensory practitioners in order to better understand the limitations and advantages of the method, compared to the traditional sensory approaches.

\section{Major results in Napping studies}

The method was first published by Pagés in 2005, but it only started to spread in the 2010s, as shown by the fact that only two of the 17 articles examined were written before 2010 . Out of the 17 articles reviewed, five focus on wines, three on beers, four on other fermented foods - yoghurt, balsamic vinegar and cheese - and one article is "mixed", dealing with yoghurt and bread. The remaining four articles deal with non-fermented foods - green tea, liver pâtés and soy milk.

Of the 17 cases examined, 11 used traditional - full - Napping, two used partial Napping in addition to full, the partial version of the study was used in a total of four articles, and the least used was grouped Napping, with only two articles. However, the complementary studies were much more varied: UFP and FP methods were the most common, but the use of CP and hedonic scales was also explicitly common.

As can be seen in Table 1, wines were the least likely to have been evaluated by naïve panelists (consumers), while in the case of non-fermented foods they were more involved. There was only one article that used all three types of panelists, that was Torri's 2013 article comparing the perceptions of different panelists.

It can also be observed from the data in Table 1 that the three types of assessors were used in almost equal proportions when analyzing beers. This may be explained by the relatively late start of the method on beers - the first paper on Napping in beers was published in 2013. A further explanation may be that wines are typically esteemed as 'higher order' drinks (Charters, 2006), so a more qualified assessor and a more accurate description is expected.

In the case of other fermented products and non-fermented products, it is important to highlight that no industry experts were involved in any of the studies, which may be explained

Table 1. Distribution of panelist types for each product category in the analyzed articles

\begin{tabular}{lcccc}
\hline & \multicolumn{3}{c}{ Number of articles } \\
\cline { 2 - 5 } Product type/Panelist type & Trained panelist & Industry expert & Naive & Sum. \\
\hline Beer & 1 & 1 & 2 & $\mathbf{3}$ \\
Wine & 3 & 3 & 1 & $\mathbf{5}$ \\
Other fermented products & 2 & 0 & 3 & $\mathbf{5}$ \\
Other & 1 & 0 & 3 & $\mathbf{4}$ \\
\hline
\end{tabular}


by the fact that for the majority of those products, industry experts are not typically skilled in tasting (except perhaps green tea and balsamic vinegar).

As a case study, we present the research of Luisa Torri et al. (2013), who investigated the extent to which people at different levels of training can differentiate between wines. The study involved 9 trained panelists, 13 wine experts and 81 consumers. The trained panelists and winemakers also performed traditional profiling, which consisted of four stages and was repeated four times. The test was conducted using traditional Napping. One of the 11 samples was given to the assessors in duplicate to check their accuracy. After the Napping procedure, the samples had to be ranked on a 9-point scale based on perceived quality, where 1 indicated very poor quality and 9 indicated excellent quality. Finally, panels of trained assessors and industry experts were asked to rank the samples on a scale of how good they thought the sample was as an example of the wine variety (Tuscan Sangiovese) that was the focus of the research, where 1 - very poor example, 9 - excellent example. Consumers were asked to complete a socio-cognitive test measuring involvement and innovativeness, followed by traditional Napping. After the Napping test, they had to rank the samples on a hedonic scale ranging from 0 to 100 . The data were analyzed by pooling the scores of trained assessors and industry experts and comparing them with the scores of consumers, with a significant difference between the two groups as shown in Fig. 1. Assessors with higher level of expertise were able to make more distinction among the samples. Consumers' data shows that the position of samples are much closer to each other.

\section{DISCUSSION}

Napping is a rapid sensory evaluation method, in which the assessor takes a holistic approach to evaluating samples rather than the descriptive methods. Panelists have to sort samples on the table according to their similarity or dissimilarity to each other, based on their own criteria
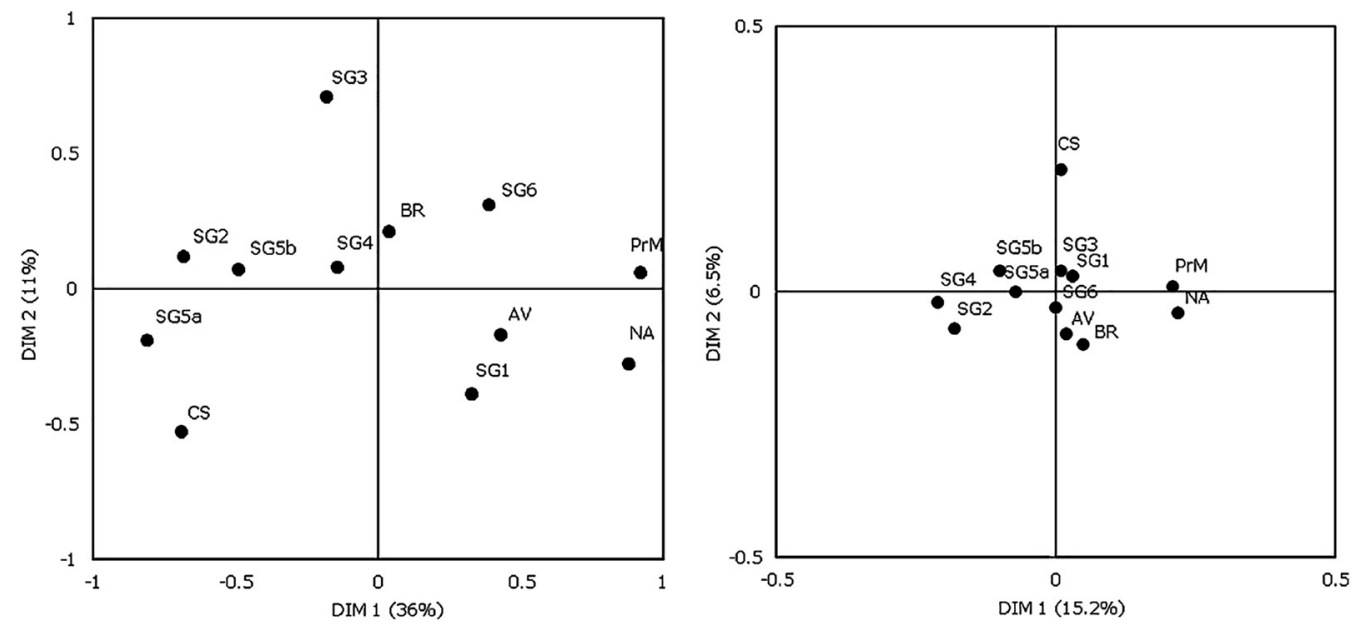

Fig. 1. Pooled Napping results of trained assessors and winemakers (left) and Napping results of consumers (right) (Torri et al., 2013) 
which they do not specify, by placing similar samples closer together and dissimilar samples further apart. Napping might be performed in 3 major types:

- Traditional/Full Napping, where the assessors evaluate the samples holistically,

- Partial Napping, where the assessor evaluates one attribute at a time, the test is typically repeated several times, focusing on different attributes, thus providing a more accurate characterization than the traditional version,

- Sorted Napping, where after traditional Napping the assessor groups the samples into groups, the result is even closer to the individual assessment criteria than in traditional Napping.

Since Napping essentially does not involve any descriptive steps, but only observes differences and similarities between samples, it is often combined with other sensory methods that are descriptive

- Flash Profile: an accelerated form of traditional profiling, with fewer steps and simpler methods of assessment,

- Ultra Flash Profile: after Napping, the assessor writes comments next to the position of the samples, this approach is the most often used to complement Napping tests,

- Hedonic scales: shows consumer preference, thus provides important feedback for marketing, - CATA/RATA: facilitates the involvement of untrained panelists, as they do not have to rely on their own terms. It is also easier to evaluate than traditional methods, but loses the individual aspect that is the core value of Napping,

- Traditional profiling: carried out by experts, generally the most accepted method due to its precision, but long and difficult to carry out.

This last statement is supported by the results of the 2008 study by Perrin et al., in which they concluded that a combination of Napping and UFP cannot provide data with the same level of precision as traditional profiling but can identify significant differences. Traditional Napping can be used mainly for differentiation, while partial Napping with UFP can be used for description. Based on other research, e.g., Ross et al. (2012), it can be concluded that for very similar samples, it is important to perform assessments in more than two replicates to get an accurate picture when analyzing Napping data. Based on the research of Torri et al. (2017), it can be stated that using combined methods, it was possible to map well the similarities and differences between the two groups of consumers.

For all sensory methods, an important factor is the duration and resource requirements of the panel training and screening needed for the implementation. Preliminary training had a positive impact on the results of the Napping tests. The product training had the most positive effect on the test, i.e., the assessors who attended the product training were the most accurate at matching samples enriched with the same flavoring and were able to detect differences in their intensity. Furthermore, an important finding was that reproducibility was also improved by the pre-training sessions. These results suggest that, even with rapid methods, the reliability of results can be increased by taking the time to gain some level of sensory familiarity with the product group under investigation. Using a methodology similar to that described in the 2016 study by Liu et al., Napping is also more applicable to consumer tests. In terms of time required for the test, it is therefore a better decision to provide a preliminary, shorter product-specific training rather than to repeat the session to investigate reproducibility. The basic principle of this method is primarily to distinguish between samples and to characterize them, rather than to 
record exact figures, so that the classical reproducibility criteria should be given a different weight.

To determine the applicability of Napping, it was important to consider the 2013 research by Torri et al., who found that trained panelists and industry experts are able to identify similarities and differences with greater accuracy than consumers. In their opinion, the former two groups compare samples to a memorized "wine prototype" that they recall from previously experienced wines. Furthermore, an important finding was that, in general, high-engagement and innovative consumers were able to differentiate more accurately based on the tests. It is also important to point out that those consumers who are more familiar with the product being tested will be able to give a more accurate description of the product in the additional tests, but this does not mean that they will be able to differentiate the samples better.

\section{CONCLUSIONS}

The field of sensory testing is constantly evolving. Many methods are being developed, some of which will remain part of research procedures for a long time. The Napping method has been in use for a relatively short time, so it is not yet possible to say exactly how well it will be accepted and established. The analysis suggests that researchers and practitioners who see the product from a holistic perspective and understand the value of Napping's results and are able to translate its outputs into practice will be more open to utilize this process. Another factor may be the extent to which Napping is adopted as a method by mainstream sensory software systems and whether it will be included in the procedures described by ISO standards. Currently Napping is a possible choice for those researchers who would like to integrate a rapid sensory evaluation technique in their study without the time consuming panelist selection and training phase.

\section{ACKNOWLEDGEMENTS}

The Authors thank the support of the grant EFOP-3.6.3-VEKOP-16-2017-00005.

\section{REFERENCES}

Abdi, H. and Valentin, D. (2007). Multiple factor analysis (MFA). Encyclopedia of Measurement and Statistics: $1-14$.

Charters, S. (2006). Wine and society: the social and cultural context of a drink. Routledge, 88: 310.

Escofier, B. and Pages, J. (1994). Multiple factor analysis (AFMULT package). Computational Statistics \& Data Analysis, 18(1): 121-140.

Liu, J., Grønbeck, M. S., Di Monaco, R., Giacalone, D., and Bredie, W. L. (2016). Performance of Flash

Profile and Napping with and without training for describing small sensory differences in a model wine.

Food Quality and Preference, 48: 41-49.

Pagès, J. (2003). Recueil direct de distances sensorielles: Application à l'évaluation de dix vins blancs du

Val-de-Loire. Sciences des aliments, 23(5-6): 679-688. 
Pagès, J. (2005). Collection and analysis of perceived product inter-distances using multiple factor analysis: Application to the study of 10 white wines from the Loire Valley. Food Quality and Preference, 16(7): 642-649.

Perrin, L., Symoneaux, R., Maitre, I., Asselin, C., Jourjon, F., and Pagès, J. (2008). Comparison of three sensory methods for use with the Napping ${ }^{\circledR}$ procedure: case of ten wines from Loire valley. Food Quality and Preference, 19(1): 1-11.

Risvik, E., McEwan, J. A., Colwill, J. S., Rogers, R., and Lyon, D. H. (1994). Projective mapping: a tool for sensory analysis and consumer research. Food Quality and Preference, 5(4): 263-269.

Ross, C. F., Weller, K. M., and Alldredge, J. R. (2012). Impact of serving temperature on sensory properties of red wine as evaluated using projective mapping by a trained panel. Journal of Sensory Studies, 27(6): 463-470.

Torri, L., Dinnella, C., Recchia, A., Naes, T., Tuorila, H., and Monteleone, E. (2013). Projective mapping for interpreting wine aroma differences as perceived by naïve and experienced assessors. Food Quality and Preference, 29(1): 6-15.

Torri, L., Jeon, S. Y., Piochi, M., Morini, G., and Kim, K. O. (2017). Consumer perception of balsamic vinegar: a cross-cultural study between Korea and Italy. Food Research International, 91: 148-160.

Open Access. This is an open-access article distributed under the terms of the Creative Commons Attribution 4.0 International License (https://creativecommons.org/licenses/by/4.0/), which permits unrestricted use, distribution, and reproduction in any medium, provided the original author and source are credited, a link to the CC License is provided, and changes - if any - are indicated. (SID_1) 